\section{6 \\ SORVEGLIANZA DELLE INFEZIONI OSPEDALIERE IN UN REPARTO DI CHIRURGIA: REVISIONE DEI RISULTATI DEGLI ANNI 2005 E 2006}

Gualdi P.', Collini L.', Schinella M.', Stefenelli N.', Pasqualini A. ${ }^{3}$, Mariotti G. ${ }^{4}$

'U.O. Patologia Clinica,

2U.O. Chirurgia,

3U.O. Farmacia,

${ }^{4}$ Direzione Medica; Ospedale S. Maria del Carmine

- P.le S. Maria 6, 38068 Rovereto (TN)

Introduzione. Il problema delle infezioni nosocomiali rimane un aspetto rilevante nell'attuale gestione dell'assistenza sanitaria nonostante le notevoli acquisizioni nella conoscenza dei fattori di rischio e nell'utilizzo di numerosi interventi di prevenzione. Il sistema di rilevazione tramite sorveglianza attiva delle infezioni nosocomiali, utilizzato nel nostro ospedale e gestito dal laboratorio, ha consentito di raccogliere i dati necessari a rilevare le Infezioni Ospedaliere (I.O.) nel reparto di Chirurgia nel periodo 2005-2006.

Metodi. Tramite lo strumento informatico VIGI@ct (bioMerieux) sono stati raccolti i dati riferibili agli anni 2005 e 2006 relativi all’Unità Operativa di Chirurgia che partecipa al progetto di sorveglianza delle I.O. del nostro ospedale. Si tratta di un rilevamento costante e quotidiano che prevede la segnalazione di presunte I.O. basata su dati di laboratorio ed una successiva conferma da parte del clinico tramite la compilazione di un questionario. In un secondo momento un gruppo di lavoro del Comitato per le Infezioni Ospedaliere, costituito da un microbiologo, un farmacista e un chirurgo, ha attuato una revisione critica delle cartelle cliniche dei pazienti con I.O. confermata allo scopo di controllarne l'origine, la profilassi pre-operatoria e la terapia antibiotica attuata.

Risultati. Nel 2005 e nel 2006 sono state segnalate rispettivamente 30 e 28 infezioni definite come ospedaliere al momento della compilazione del questionario che settimanalmente viene consegnato al clinico di reparto. Si tratta di infezioni delle vie urinarie in pazienti portatori di catetere vescicale, sepsi e sepsi CVC correlate, infezioni della ferita chirurgica o addominali post-intervento. Altre, al momento di un attenta revisione delle cartelle cliniche, sono state invece ridefinite come infezioni endogene oppure comunitarie.

Conclusioni. Le infezioni nosocomiali restano un problema frequente e grave nella pianificazione dell'assistenza sanitaria. Un programma di sorveglianza ed una successiva revisione dei dati tramite cartella clinica riesce ad arginare il trend di prevalente aumento. Tuttavia le pratiche assistenziali da adottare per il controllo delle infezioni ospedaliere rappresentano a tutt'oggi una tematica ancora aperta che nell'ambito ospedaliero abbraccia non solo più figure professionali ma anche un gran numero di misure preventive che le stesse sarebbero tenute ad adottare.

\section{7 \\ SORVEGLIANZA ATTIVA IN ICU: FATTORI DI RISCHIO PER ACQUISIZIONE DI MRSA E CARATTERIZZAZIONE DEI CEPPI ISOLATI}

\author{
Malandrin S. M. I., Montesissa L., Cocca G.,Vella P., \\ Grosso S., Saudelli M. \\ A. O. Fatebenefratelli e Oftalmico, C.so P.ta Nuova 23,
20124 Milano
}

Introduzione. In questo studio si è cercato di definire a livello locale la dinamica attraverso cui i pazienti ricoverati in ICU divengono portatori o infetti da S. aureus meticillino resistente (MRSA), il tasso con cui ciò si verifica, i fattori di rischio e le condizioni fisiopatologiche che accompagnano o favoriscono questo evento. Dei ceppi di MRSA isolati si è poi stilato un profilo di resistenza a diversi antibiotici al fine di caratterizzare i ceppi di origine comunitaria o nosocomiale.

Metodi. Da febbraio a maggio 2007 presso l’A. O. Fatebenefratelli e Oftalmico di Milano è stata condotta una sorveglianza attiva dei pazienti ricoverati presso le UUOO di Anestesia e Rianimazione (SAR) e Medicina d'Urgenza (MedUrg). Si sono inclusi nello studio solo pazienti rimasti in Reparto almeno 48 ore. I dati demografici, i fattori di rischio, l'anamnesi patologica prossima e remota, la terapia antibiotica e gli eventuali interventi chirurgici sono stati registrati su apposita scheda statistica computerizzata. Lo stato di colonizzazione da MRSA del paziente è stato sorvegliato mediante tamponi nasali all'ingresso in Reparto, poi settimanalmente e all'uscita dal Reparto. Lo stato di infezione è stato ricercato mediante analisi delle cartelle cliniche, interviste ai medici curanti, i dati biochimici e microbiologici dei campioni inviati in Laboratorio. I saggi degli antibiotici sono stati eseguiti mediante diluizione in brodo in micropiastra o mediante E-Test, a seconda dell'antibiotico saggiato.

Risultati. In 4 mesi sono stati arruolati nello studio 233 pazienti, di cui 124 femmine (53,2\%), 109 maschi (46,8\%) con età media di 69,3 anni mediana di 76; 158 ricoverati in MedUrg e 75 in SAR. Sono stati identificati 30/233 (12,8\%) pazienti portatori di MRSA: 17/30 pazienti (56\%) erano portatori già all'ingresso in Reparto, mentre 13/30 (44\%) lo hanno acquisito durante la degenza; 5/233 pazienti $(2,1 \%)$ hanno sviluppato un'infezione: 3 casi di HAP, 1 HAP + sepsi e 1 sepsi. La lunghezza del ricovero in ICU è il principale fattore di rischio individuato per lo sviluppo dello stato di portatore $(\mathrm{P}<0,01)$, l'utilizzo di alcuni devices invasivi (CVC e catetere urinario) mostrano una correlazione meno forte $(\mathrm{P} \approx 0,01)$, mentre nel nostro studio sembrano aver meno peso l'età dei pazienti ed il Reparto in cui sono ricoverati

Conclusioni. La sorveglianza dei pazienti ricoverati in Reparti di cura intensiva ha permesso di individuare alcuni fattori di rischio per lo sviluppo di un'infezione da MRSA. La sorveglianza attiva, sebbene impegnativa da un punto di vista organizzativo, dimostra una buona sensibilità nell'individuare i pazienti portatori e un'ottima specificità (sensib.=90\%; specif.=100\%). 